\title{
Coral Platform: The Smart Startup Ecosystem Tool
}

\author{
Rafael Escalfoni ${ }^{1,2}$, Leonardo Marinho ${ }^{1}$, Camila Lacerda ${ }^{1}$, \\ Rodrigo Oliveira $^{1}$, Kesia Mamede ${ }^{1}$, Mônica F. da Silva ${ }^{1}$, Jonice Oliveira ${ }^{1}$ \\ ${ }^{1}$ PPGI - Universidade Federal do Rio de Janeiro (UFRJ) \\ Rua Athos da Silveira, s n - 20230-030 - Rio de Janeiro - RJ - Brazil \\ ${ }^{2}$ CEFET/RJ - Campus Nova Friburgo \\ Av. Gov. R. Silveira, 1900 - 26500-000 - Nova Friburgo - RJ - Brazil \\ rafael.escalfoni@cefet-rj.br, \{leonardomarinho.nf, camilalacerda 8 \\ roliveira, kmamede, mfsilvamail\}@gmail.com, jonice@dcc.ufrj.br
}

\begin{abstract}
A smart startup ecosystem must own capabilities and information to the accurate allocation of resources among its participants. So, it uses the synergy of partnerships to enhance startups and boost new ventures. The constant evolution of such communities implies an exponential increase in produced data, which can bring essential business insights, despite the involved technical challenges, such as diverse sources, structures, and meanings. To tackle these issues, we are developing the Coral Platform, that joins a repository of data collectors and preprocessors, a flexible dataset, graph models, and ways of visualization.
\end{abstract}

\section{Introduction}

A smart startup ecosystem must own capabilities and enough information to the accurate allocation of resources, costs, and benefits among its participants to promote economic growth and technological development [Chourabi et al. 2012]. Most of such assets are shared through the dynamics of startup ecosystem relationships, that also set the synergy needed to sustain technical advances, new problem insights, and tacit information gained from informal learning processes [Giustiniano and D'Alise 2015]. The network structure may imply differential advantages or restrictions for the actors. Thereby, community evolution analysis indicates predicting and understanding changes in the distribution of benefits and constraints on the network [Ahuja et al. 2012].

Furthermore, the complexity and constant evolution of entrepreneurial communities have produced an exponential increase in data [Hashem et al. 2016]. This enormous volume of data collected from different sources, with multiple structures and formats, offers a high possibility of obtaining valuable information if supported by appropriate analysis and visualization tools [Chourabi et al. 2012]. However, the most common strategies for analyzing the relationship with the business groups are based on the collection of a single data source from dominant organizations or obtained by surveys applied in the community. These approaches ignore that there are a large number of activities that take place at the individual and corporate levels [Basole et al. 2016]. As a result, meaningful information about the community is lost, as informal aspects, as social and cultural data.

Thereby, we propose the Coral Platform for promoting an understanding of entrepreneurial communities, revealing tacit social and technical ties. For this, it collects data from different sources, such as social media, business portals, and technical datasets, 


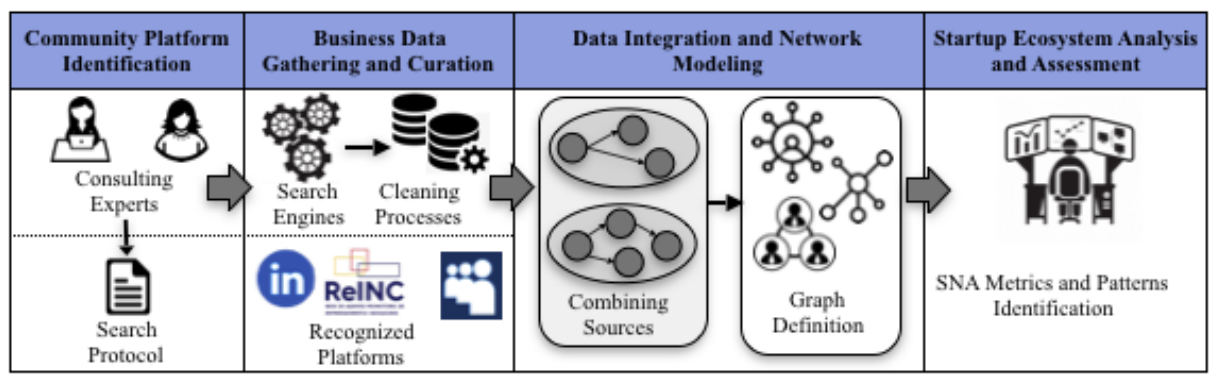

Figure 1. Coral Framework

and offers visualization and diagnosis through a set of indicators based on social network analysis (SNA). The tool consists of a set of extensible modules to allow adaptation according to the data availability and the desired analyzes.

\section{Related Works}

There are some considerable advances in the study of collaborative networks for entrepreneurial communities. [Basole et al. 2016] presented a tool to identify the segment, the total number of partner collaboration, the number of collaborations, the specialties, and the trusting level in a business ecosystem. The graph visualization model supports the intelligent management of partnerships and decision-making. They did not address the particularities of startup ecosystems, but the issues raised in these studies elucidate important points about the relationships and the impact of interactions. In turn, [Luz et al. 2018] introduced a sustainability model for small-to-medium enterprise software ecosystems, which contains startups while [Faber et al. 2018] highlighted the importance of the entrepreneurial ecosystem for the economic development of smart cities and showed a robust visualization model to represent the different relationships existing in the environment.

One of the main aspects that distinguish these approaches is the data analysis strategy because they did not present specific metrics to analyze the relations in their method. Besides, our smart gathering is automatic and passive, using all available public data on the internet. Thus, the Coral retrieve procedure does not overload the stakeholders with new tasks, such as fill forms or answer surveys. This is important since it can avoid interferences that could compromise the data accuracy.

\section{Features}

The Coral Platform allows adaptations according to the availability of data and the intended studies. Its modules support a framework designed to process and store raw data, configure graphs and SNA indicators, and display them to startup ecosystem stakeholders, described in Figure 1 [Escalfoni et al. 2020]. The process begins with consulting experts to determine the data sources. Then, the system provides settable search engines to collect and structure data. After that, it supports the entities and relationships characterization to build graphs that will be used for community analysis, through the indicators to support the investigation process, as shown in Figure 2. The indicators are SNA-based heuristics and aim to give meaning to the collected data. The researchers make their definition during study planning. In our example, we used ranking, abilities, reputation, and relevance using datasets from the LinkedIn, Lattes, and ReINC ${ }^{1}$.

\footnotetext{
${ }^{1}$ ReINC is a Network of Promoters of Innovative Enterprises of Rio de Janeiro
} 


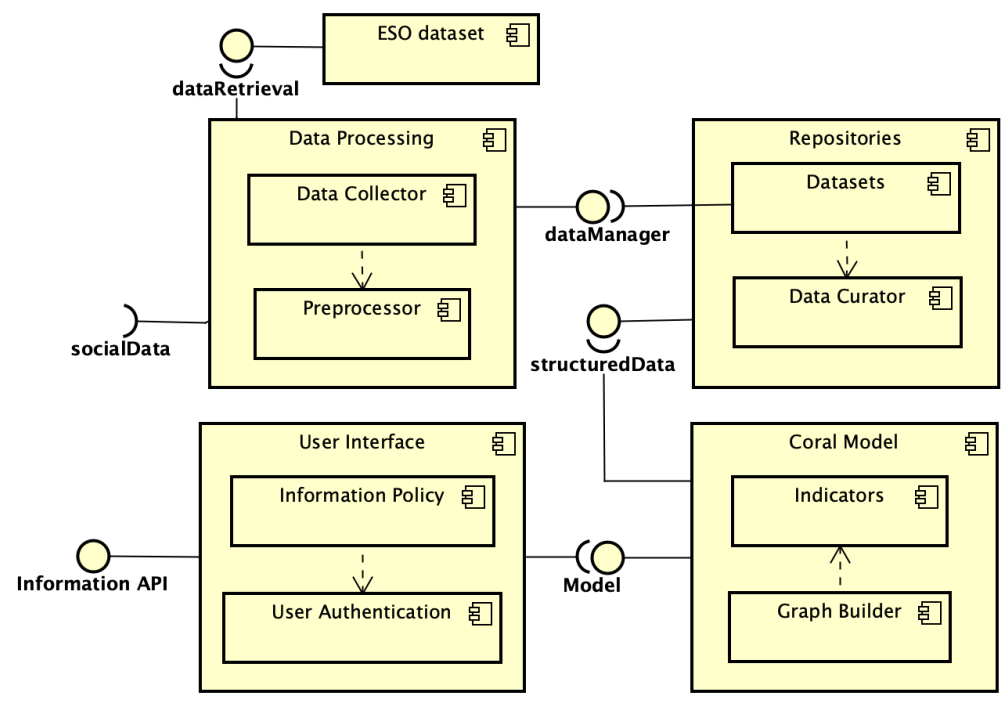

Figure 2. Architecture of Coral Platform

The system has two distinct actors: the Researcher, responsible for setting up the platform, data gathering and definition of the indicators and graphs; and the Stakeholder, a business specialist that specifies research objectives, and points out data sources and drives the meaning of gotten information. The Stakeholder is the end-user, having access to dynamic graphs, and final reports. The main features are detailed bellow:

- Collect Social Data: Social platforms have specific interaction features that require distinct gathering strategies. The use case starts with determining social media attributes, such as recommendations, likes, comments, responses. After that, the system offers a collector configuration to adjust gathering scripts.

- Collect Business Data: Business sites provide information about companies, their products, services, and technical details. They often offer APIs for data collection, which only requires setting up the structure of the data file. When there is no API, it is necessary to adopt one of the collector scripts available in the module.

- Setup Graphs: Before to analyze networks, the graph definition relies mainly on the available data. The system displays all entities and critical attributes identified in the database. For each graph, the Researcher must indicate which elements are network nodes and what are their relationships. Finally, the tool requests to choose the SNA indicators, explaining the available metrics.

- Plot Graphs: When the environment is ready, the system renders dynamic graphs that help in the analysis of the community aspects defined previously. The system has an interface where the networks are displayed.

- Detail Organization: When a graph is plotted, the system allows choosing one of the drawn nodes to show the metrics applied to it, as shown in Figure 3. It is an extension of the Plot Graph feature and displays the defined indicators.

\section{Conclusions}

We presented a web tool that joins a set of configurable artifacts to monitor sociotechnical aspects in startup ecosystems. It is an ongoing version, in a project based on 


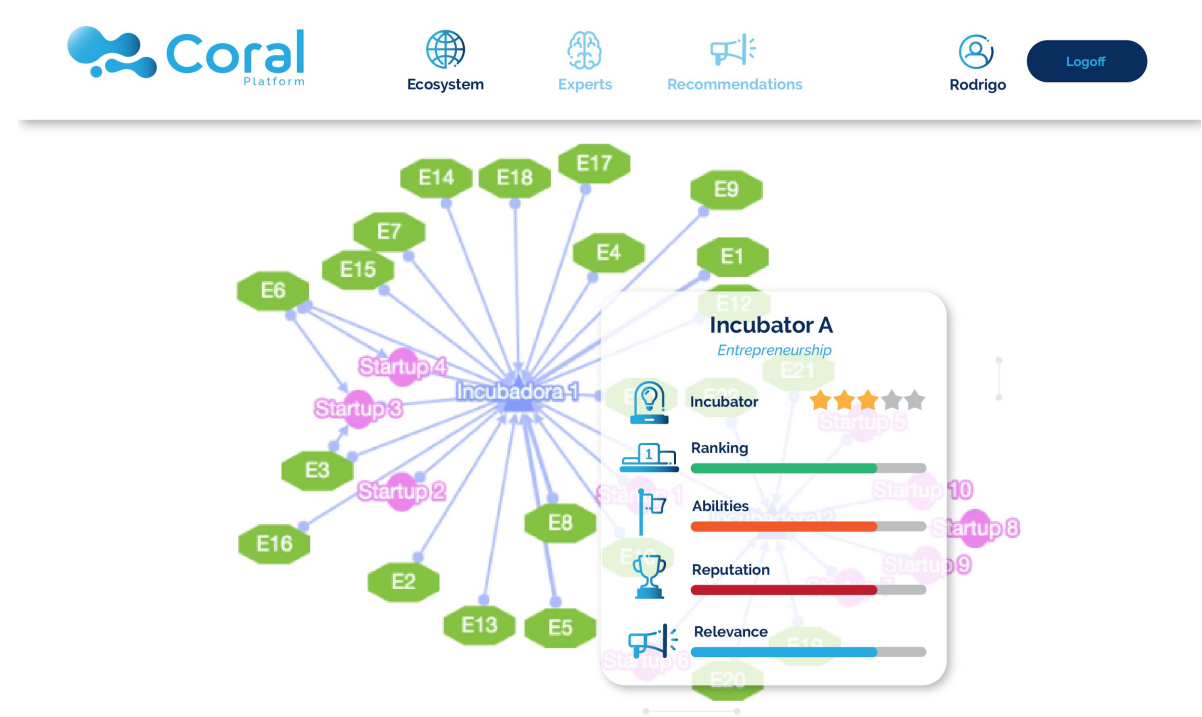

Figure 3. Detail Organization

SCRUM, that has the main functionalities implemented. The current release is available at http://bit.do/frr2a and it is running at http://bit.do/frr4P. A video clip demoing the tool can be found at https://youtu.be/J07UN8FZdnc. The tool was tested in [Escalfoni et al. 2020] and is being improved the usability and implementing new crawlers.

\section{References}

Ahuja, G., Soda, G., and Zaheer, A. (2012). The genesis and dynamics of organizational networks. Organization Science, 23(2):434-448.

Basole, R. C., Huhtamaki, J., Still, K., and Russel, M. G. (2016). Visual decision support for business ecosystem analysis. Expert Systems With Applications, 65:271-282.

Chourabi, H., Nam, T., Walker, S., Gil-Garcia, J. R., Mellouli, S., Nahon, K., Pardo, T. A., and Scholl, H. J. (2012). Understanding smart cities: An integrative framework. In 45th HICSS, pages 2289-2297, Hawaii. IEEE.

Escalfoni, R., Silva, M. F., and Oliveira, J. (2020). Visualizing social relations in startup ecosystems using the coral platform. In SBSI, São Bernardo do Campo. ACM.

Faber, A., Hernanderz-Mendez, A., Rehm, S.-V., and Matthes, F. (2018). An agile framework for modeling smart city business ecosystems. In XX ICEIS 2018, volume 2, pages 39-50, Funchal. ACM.

Giustiniano, L. and D'Alise, C. (2015). Networks, clusters, and small worlds - are they related? Journal of Organization Design, 4(2):48-53.

Hashem, I. A. T., Anuar, N. B., and Adewole, K. S. (2016). The role of big data in smart city. International Journal of Information Management, 36:748-758.

Luz, P. B. V., Fernandes, J., Valença, G., and Santos, R. P. (2018). An exploratory study on the sustainability of emerging small-to-medium enterprises ecosystems. In IX WAIHCWS, pages 95-104, Belém. SBC. 